\title{
Effect of incubation in simulated body fluid on dielectric and photoluminescence properties of nano-hydroxyapatite ceramic doped with strontium ions
}

\author{
Megha P. Mahabole ${ }^{1}$, Manjushree M. Bahir ${ }^{1}$, Namdev V. Kalyankar², Rajendra S. Khairnar ${ }^{1 *}$ \\ ${ }^{1}$ School of Physical Sciences, Swami Ramanand Teerth Marathwada University, Nanded, India \\ ${ }^{2}$ Yeshwant Mahavidyalaya, Nanded, India \\ Email: ${ }^{*}$ rskhairnarsps@gmail.com
}

Received 31 January 2012; revised 12 March 2012; accepted 21 March 2012

\begin{abstract}
This paper reports the dielectric and photoluminescence measurements of ossiointegrated strontium doped hydroxyapatite bioceramic. The study on invitro bioactivity of Sr-HAp is carried out by using modified simulated body fluid. Dielectric properties of these ossiointegrated samples are studied in the frequency range $10 \mathrm{~Hz}$ to $1 \mathrm{MHz}$ at room temperature. Dielectric constant is found to be decreasing with an increase in incubation period and frequency of applied ac field. Higher the period of incubation and applied ac frequency, higher is the dielectric loss. AC conductivity shows frequency independent behaviour for all samples in lower frequency region and then increases linearly with increase in frequency. Sample incubated for longer duration shows higher conductivity. The optical properties of incubated Sr-HAp samples are also investigated with photoluminescence (PL) technique. The PL study shows the blue emission at around $476 \mathrm{~nm}$ when excited at 409 $\mathrm{nm}$. The PL intensity increases with increase in incubation duration and Sr-substitution.
\end{abstract}

Keywords: Hydroxyapatite; Dielectric Properties; Luminescence; Impedance; Biomedical Applications

\section{INTRODUCTION}

Hydroxyapatite bioceramic (HAp: $\left.\mathrm{Ca}_{10}\left(\mathrm{PO}_{4}\right)_{6}(\mathrm{OH})_{2}\right)$ is one of the attractive member of calcium phosphate family having same mineral composition as that of calcified tissues of vertebrates. It not only promotes the new bone formation but also attach directly to living tissue chemically via formation of biologically active apatite layer, leading to interfacial bonding [1,2]. Hence HAp has gained extensive acceptance for bone implant applica-

*Corresponding author. tions due to its excellent biocompatibility, bioactivity, non immunogenic, non inflammatory behaviour and osteoconductive nature [3-5]. Literature survey reveals that most of the research work carried out on HAp based materials is concentrated on understanding bioactive nature of HAp with the help of in-vivo and in-vitro bioactivity tests [6-9]. Besides this, HAp is widely used in other application areas, which are basically based on electrical behaviour of HAp, such as chemical sensor [10-12], ionexchanger [13-16], fuel cell [17]. Recently, HAp is being used for electrical applications such as an electrical insulating coating for electronic devices including implantable devices and dielectric coating [18]. It is reported that the electrical and dielectric properties are useful for biomaterial applications since the bone is natural composite made up of fluids, collagen and HAp matrix [19]. Hence it is worth to carry out study on electric and dielectric properties of HAp to have better understanding of electrical and biological phenomena. From this point of view, complex impedance spectroscopy technique is an appropriate approach to investigate the electrical properties of hydroxyapatite material. Few reports are available on dielectric and electric properties of HAp. These studies not only describe dielectric behaviour of pure HAp, ion exchanged HAp and HAp based composites but also dependence of dielectric constant on sintering temperature and porosity [10,18-24].

Furthermore, few reports have been focused especially on Sr-HAp because partial substitution of $\mathrm{Sr}^{2+}$ ions in HAp, with low concentration, is not only found to be beneficial in the osteoporosis treatment, dissolution behaviour and growth kinetics but also for improvement in mechanical properties. Therefore, Sr-HAp has found promising applications in bone repairing in dentistry, orthopaedic and reconstructive surgery because Sr-HAp is supposed to stimulate bone formation. Though strontium and calcium belong to same group of periodic table, the ion exchange capacity of Sr-HAp is found to be su- 
perior to HAp [25-29]. J. Bera et al. have carried out comparative study on dielectric properties of HAp and Sr-HAp in detail [30].

In addition, detailed luminescence studies on HAp doped with rare earth metals are also carried out [31-35]. Zhang et al. have studied the luminescence property of nano and microcrystalline hydroxyapatite with multiple morphologies [36]. A study on the inherent luminescence of annealed biomimetic apatite grown on $\mathrm{NaOH}$ treated titanium has been carried out by Muller et al. [37]. Chung et al. have used photoluminescence technique to show the formation of hydroxyapatite coating through a bio-mimetic process [38]. Few studies have shown that strontium doped HAp is an efficient luminescent material [39, 40]. However the photoluminescence and dielectric properties of partially substituted $\mathrm{Sr}^{2+}$ ions in HAp, after incubation in $\mathrm{m}-\mathrm{SBF}$ for particular duration, have not been carried out so far.

Hence, the present paper deals with measurement of photoluminescence and dielectric properties of incubated Sr-HAp in detail. In-vitro bioactivity study on Sr-HAp pellets is carried out by incubating them in modified SBF for various durations. The incubated Sr-HAp pellets are characterized by XRD, FTIR and SEM. The dielectric properties of these incubated ion exchanged HAp samples are studied in the frequency range of $10 \mathrm{~Hz}$ to 1 MHz. The fluorescence study is also carried out to show the development of apatite layer on Sr-HAP.

\section{MATERIAL AND METHODS}

\subsection{Hydroxyapatite Synthesis}

Nano-sized hydroxyapatite (HAp) is synthesized via a precipitation method described in previous work $[5,10]$. Di-ammonium hydrogen orthophosphate $\left(\left(\mathrm{NH}_{4}\right)_{2} \mathrm{HPO}_{4}\right)$ solution is dropped in to calcium nitrate tetrahydrate $\left(\mathrm{Ca}\left(\mathrm{NO}_{3}\right)_{2} \cdot 4 \mathrm{H}_{2} \mathrm{O}\right)$ solution to achieve necessary $\mathrm{Ca} / \mathrm{P}$ molar ratio (1.67). The precipitation reaction is carried out at $100^{\circ} \mathrm{C}$ temperature under vigorous stirring for five hours and during the process $\mathrm{pH}$ of the reaction is controlled by using ammonia solution. The obtained milky precipitate is aged for 24 hours at room temperature followed by rinsing with distilled water for the removal of ammonia. The product is then dried in an oven at $100^{\circ} \mathrm{C}$ and crushed into powder form. Dried product (HAp) is finally sintered to $1000^{\circ} \mathrm{C}$ in air atmosphere for 2 hours.

\subsection{Ion Exchange of Hydroxyapatite}

Ion exchange is carried out for partial substitution of $\mathrm{Ca}^{2+}$ ions by $\mathrm{Sr}^{2+}$ ions in sintered hydroxyapatite powder to form Sr-HAp. Ion exchange process consists of addition of known amount of HAp to an aliquot of $25 \mathrm{ml}$ of $0.005 \mathrm{M}$ strontium nitrate solution followed by five hour continuous shaking of suspension at room temperature. After achieving saturation ion exchange equilibrium, the suspension is filtered and dried using air oven at $100^{\circ} \mathrm{C}$ overnight. Ion exchanged powder (Sr-HAp) is compacted at 5 ton pressure to obtain a disc of $13 \mathrm{~mm}$ diameter with $2 \mathrm{~mm}$ thickness using hydraulic press. These pellets are then sintered at $500^{\circ} \mathrm{C}$ in air for about $2 \mathrm{hrs}$ and are used for further in-vitro bioactivity tests and electrical measurements.

\subsection{Preparation of Simulated Body Fluid (SBF)}

Modified simulated body fluid (m-SBF) is prepared using various reagents as per the ionic concentration presented in Table 1 [41,42]. It reported that deficiency in $\mathrm{HCO}_{3}^{-}$ion concentration in Kokubu SBF solution takes longer period to form calcium apatite nuclei on the HAp surface. Therefore, $\mathrm{m}-\mathrm{SBF}$ is used wherein the required $\mathrm{HCO}_{3}^{-}$ion concentration, that resembles human plasma $(27 \mathrm{mM} /$ liter), is achieved by increasing its concentration as per Tas and Jallota.

All the reagents are added in a sequential manner, as mentioned in Table 1, to the $1 \mathrm{~L}$ double distilled water which is continuously stirred at $37^{\circ} \mathrm{C}$ temperature. Tris buffer is added pinch by pinch in order to avoid formation of precipitate. While adding of all reagents, the $\mathrm{pH}$ of the m-SBF solution is monitored by $\mathrm{pH}$ meter and finally adjusted to 7.4 using $1 \mathrm{M} \mathrm{HCl}$. Ion exchanged pellets are then immersed in freshly prepared $\mathrm{m}$-SBF for variable duration of $8,16,24$ and 32 days. After particular incubation duration, mentioned above, the pellets are removed from m-SBF solution and are rinsed with distilled water. These incubated pellets are then dried at room temperature and are characterized by XRD and SEM.

\subsection{Characterization}

XRD is carried out for structural identification of HAp and incubated Sr-HAp pellets. X-ray diffraction patterns are collected using a Rigaku Miniflex X-ray diffractometer operating at $\mathrm{CuK} \alpha(\lambda=1.5405 \AA)$ radiation in the $2 \theta$ range $20^{\circ}-60^{\circ}$.

Shimadzu make FTIR spectrophotometer (Model 2000) is used for identification of functional groups of HAp sample in scanning range of $600-4000 \mathrm{~cm}^{-1}$ with a resolution of $4 \mathrm{~cm}^{-1}$.

The surface morphology and microstructure of the Sr-HAp samples, before and after incubation, is obtained by means of Scanning Electron Microscopy (JSM/JEOL6360). Elemental composition of the Sr-HAp sample, before immersing into SBF, is determined by means of EDAX and is presented in Table 2. The dielectric measurements are carried out at room temperature in the frequency range $10 \mathrm{~Hz}$ to $1 \mathrm{MHz}$ using Impedance analyzer 
Table 1. Ion concentration of human blood plasma and m-SBF.

\begin{tabular}{lcccc}
\hline Order Reagents & $\begin{array}{c}\text { Amount } \\
\left(\mathrm{gm} / \mathrm{dm}^{3}\right)\end{array}$ & Ions & $\begin{array}{c}\text { Human Blood } \\
\text { Plasma }(\mathrm{mM})\end{array}$ & $\begin{array}{c}\mathrm{m}-\mathrm{SBF} \\
(\mathrm{mM})\end{array}$ \\
\hline 1) $\mathrm{NaCl}$ & 6.547 & $\mathrm{Na}^{+}$ & 142 & 142 \\
2) $\mathrm{NaHCO}_{3}$ & 2.268 & $\mathrm{~K}^{+}$ & 5 & 5 \\
3) $\mathrm{KCl}$ & 0.373 & $\mathrm{Mg}^{2+}$ & 1.5 & 1.5 \\
4) $\mathrm{Na}_{2} \mathrm{HPO}_{4} \cdot 2 \mathrm{H}_{2} \mathrm{O}$ & 0.178 & $\mathrm{Ca}^{2+}$ & 2.5 & 2.5 \\
5) $\mathrm{MgCl}_{2} \cdot 6 \mathrm{H}_{2} \mathrm{O}$ & 0.305 & $\mathrm{Cl}^{-}$ & 103 & 125 \\
6) $1 \mathrm{M} \mathrm{HCl}^{2}$ & $5 \mathrm{ml}$ & $\mathrm{HCO}_{3}^{-}$ & 27 & 27 \\
7) $\mathrm{CaCl}_{2} \cdot 2 \mathrm{H}_{2} \mathrm{O}$ & 0.368 & $\mathrm{HPO}_{4}^{2-}$ & 1 & 1 \\
8) $\mathrm{Na}_{2} \mathrm{SO}_{4}$ & 0.071 & $\mathrm{SO}_{4}^{2-}$ & 0.5 & 0.5 \\
9) $\left(\mathrm{CH}_{2} \mathrm{OH}\right)_{3} \mathrm{CNH} \mathrm{CN}_{2}$ & 6.057 & & & \\
10) $1 \mathrm{M} \mathrm{HCl}^{2}$ & Titrate to pH 7.4 & & \\
& at $37^{\circ} \mathrm{C}$ & & & \\
\hline
\end{tabular}

Table 2. Elemental composition of Sr-HAp.

\begin{tabular}{ccc}
\hline \multicolumn{3}{c}{ Elemental Composition of Sr-HAp } \\
\hline Element & $\mathrm{wt} \%$ & at $\%$ \\
\hline O K & 32.70 & 53.36 \\
P K & 19.39 & 16.35 \\
Ca K & 45.31 & 29.52 \\
Sr K & 2.60 & 0.77 \\
Total & 100.00 & 100.00 \\
\hline
\end{tabular}

(Quad Tech 7600). For the electrical characterization, good electrical contact with the sample is obtained by coating silver paste on both sides of the sintered pellet surfaces. Dielectric constant is measured using the formula [10]:

$$
\varepsilon^{\prime}=\frac{C d}{\varepsilon_{0} A}
$$

where $C$ is the capacitance of pellet, $d$ is the thickness of pellet, $\varepsilon_{0}$ is the permittivity of free space $\left(8.85 \times 10^{-12}\right.$ $\mathrm{F} \cdot \mathrm{m}^{-1}$ ) and $A$ is the area of the pellet.

AC conductivity of HAp and Sr-HAp is estimated by using real and imaginary parts of complex impedance measurements prior to incubation and also after incubation in $\mathrm{m}-\mathrm{SBF}$. The AC conductivity is calculated using the formula [43]:

$$
\sigma=\frac{Z^{\prime}}{Z^{\prime 2}+Z^{\prime \prime 2}} \cdot \frac{t}{A}
$$

where $Z^{\prime}$ and $Z^{\prime \prime}$ are the real and imaginary parts of the impedance, $A$ is the area of sample and $t$ is the thickness of sample.
The relaxation time $\tau$ is also calculated from complex impedance plot using the formula:

$$
\tau=\frac{1}{2 \pi f c}
$$

where $f_{c}$ is the relaxation frequency corresponds to the maximum value of $Z^{\prime \prime}$.

The bulk resistance is determined from the intercept of the each semicircular arc on the real axis and the corresponding capacitances $\left(C_{b}\right)$ are also calculated.

Fluorescence spectra of pure HAp, incubated Sr-HAp samples, and Sr-HAp sample without incubation are also obtained using Shimadzu make Spectroflurophotometer (RF5301PC). The PL measurements are carried out at room temperature in air atmosphere. A xenon lamp is used as an excitation light source. Initially, the excitation wavelength for Sr-HAp is scanned from $350 \mathrm{~nm}$ to 650 $\mathrm{nm}$ range. The excitation wavelength is chosen according to the excitation spectrum wherein the most intense excitation peak is located at $409 \mathrm{~nm}$. The emission spectra for all samples are obtained in the wavelength range 409 $\mathrm{nm}$ to $650 \mathrm{~nm}$.

\section{RESULTS}

Figure 1 shows the XRD patterns of pure HAp and incubated Sr-HAp samples. X-ray diffraction phase analysis of HAp sample, presented in Figure 1(a), shows the development of hexagonal hydroxyapatite phase. The XRD profiles of incubated Sr-HAp samples show that hydroxyapatite structure is retained by all samples during incubation in $\mathrm{m}-\mathrm{SBF}$. This indicates that replacement of $\mathrm{Ca}^{2+}$ ions by $\mathrm{Sr}^{2+}$ ions does not change the structure. However, it is observed that majority apatite peaks are slightly shifted towards higher $2 \theta$ values in all incubated Sr-HAp samples. This is attributed to lattice expansion due to higher atomic radius of $\mathrm{Sr}^{2+}$ ions than $\mathrm{Ca}^{2+}$ ions. Crystal sizes of incubated Sr-HAp samples, calculated using the Scherrer's equation, are found to be in the range $18-24 \mathrm{~nm}$.

Typical FTIR spectra of pure and Sr-HAp without incubation and incubated Sr-HAp are presented in Figure 2. The FTIR spectra show that all samples exhibit a similar kind of spectrum. The typical characteristic bands of hydroxyapatite are observed to be present. Three absorption bands present at around $1030 \mathrm{~cm}^{-1}, 1091 \mathrm{~cm}^{-1}$ and $967 \mathrm{~cm}^{-1}$ corresponds to stretching mode of characteristic phosphate group. The band at $3570 \mathrm{~cm}^{-1}$ belongs to the vibrational mode of $(\mathrm{OH})^{-}$ion. The band at 672 can be attributed to hydroxyl group. The presence of these functional groups confirms the formation of hydroxyapatite structure. The 1440 and $880 \mathrm{~cm}^{-1}$ peaks are due to $\left(\mathrm{CO}_{3}\right)^{2-}$ group which indicates that a carbonate substitution has taken place in the product during growth process. 


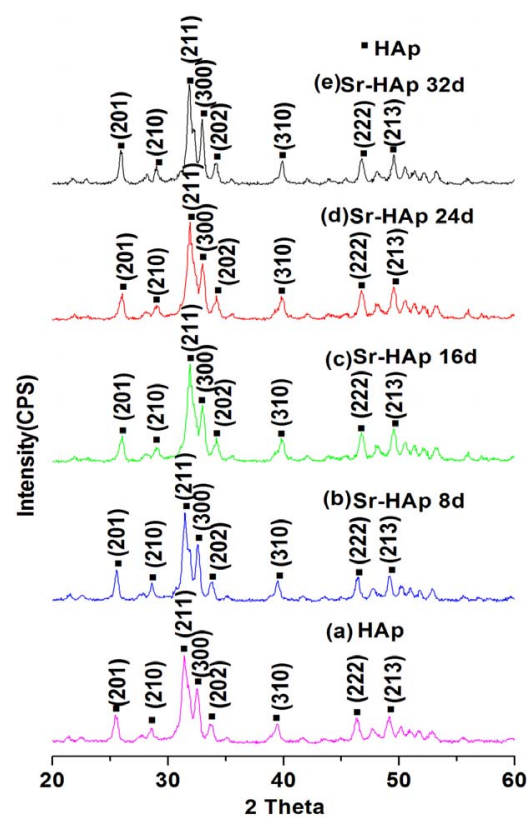

Figure 1. (a)-(e) XRD patterns of pure HAp and Sr-HAp samples after soaking in $\mathrm{m}$-SBF for variable duration.

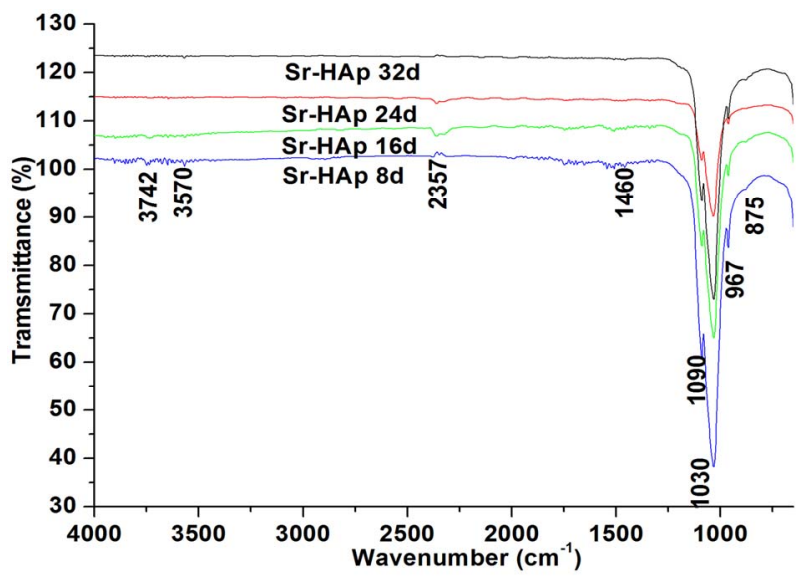

Figure 2. FTIR of incubated Sr-HAp samples showing the presence of characteristic functional groups.

Figures 3(a)-(e) depicts the surface morphologies of Sr-HAp pellets before and after incubation. The surface morphology of Sr-HAp before incubation shows the presence of small sized but separated particles (Figure 1(a)). After 8 days of incubation, nucleation of abundant tiny granular apatite particles along with pores has occurred on the surface and is depicted in Figure 3(b). The rate of apatite nucleation and porosity increases as the incubation time increases. The surface is found to be completely covered by aggregated apatite after incubation of 16 days as seen from Figure 3(c). With further increase in incubation time up to 24 days, no significant changes in surface microstructure are observed (Figure 3(d)). Figure 3(e) shows agglomeration of aggregated apatite particles and increase in porosity for the samples incubated for 32 days in m-SBF. Thus, it is concluded that incubated Sr-HAp sample shows the ability to act as a template for growth of apatite on its surface which in turn gives the evidence for the bioactive nature of SrHAp. EDAX result indicates the development of calcium and phosphorus-rich apatite phase with a lower concentration of strontium as depicted by Table 2 .

Figure 4(a) presents the change in dielectric constant as a function of frequency of applied ac field. It can be concluded that the dielectric constant decreases with increasing frequency for all the samples. The dielectric constant of Sr-HAp (82) is greater than that for pure HAp (36) (Inset in Figure 4(a)). This increase in dielectric constant is due to inclusion of $\mathrm{Sr}^{2+}$ ions in HAp matrix. This result is compatible with the values reported in the literature $[10,43]$. Dielectric constant is also observed to be dependent on incubation period. Dielectric constant, calculated at a lower frequency of $10 \mathrm{~Hz}$, is found extremely high (11000) for 8 days of incubation and is observed to be decreasing with increase in incubation duration from 16 to 32 days (5000). This may due to apatite growth on the HAp surface after soaking in m-SBF.

Typical variation in dielectric loss with frequency is presented in Figure 4(b). The dielectric loss is found to be decreasing with increase in frequency. Moreover, it is concluded that higher the period of incubation, higher is the dielectric loss.

Figure 4(c) shows the variation in real part of AC conductivity as a function applied frequency for all samples at room temperature. AC conductivity remains constant in the lower frequency region upto $1 \mathrm{KHz}$ (frequency independent) for almost all samples at RT and further increase in frequency shows linear increase in conductivity in accordance with the universal power law $\left[\sigma(\omega) \alpha \omega^{\prime \prime}\right]$ as reported earlier [44]. It is observed that larger the soaking period of the sample in $\mathrm{m}-\mathrm{SBF}$, higher is the conductivity. Therefore, the sample incubated for 32 days shows highest conductivity hence higher number of free dipoles [45]. The Cole-Cole plot (Figure 5(b)) shows the higher resistance values for lower frequencies. Therefore, at lower frequencies upto $1 \mathrm{KHz}$, the $\mathrm{AC}$ conductivity is observed to be very small as depicted in the Figure 4(c). However, for higher frequencies the resistance goes on decreasing and results in increment in conductivity. A similar kind of trend is observed in all incubated samples.

Figure 5(a) presents typical Cole-Cole plots of HAp and Sr-HAp before incubation. In case of HAp the resistance becomes so large that arc remains undefined and hence sample does not possess relaxation mechanism in the frequency range upto $1 \mathrm{MHz}$. However, addition of $\mathrm{Sr}$ in HAp matrix leads to decrease in resistance and improvement in conductivity. 


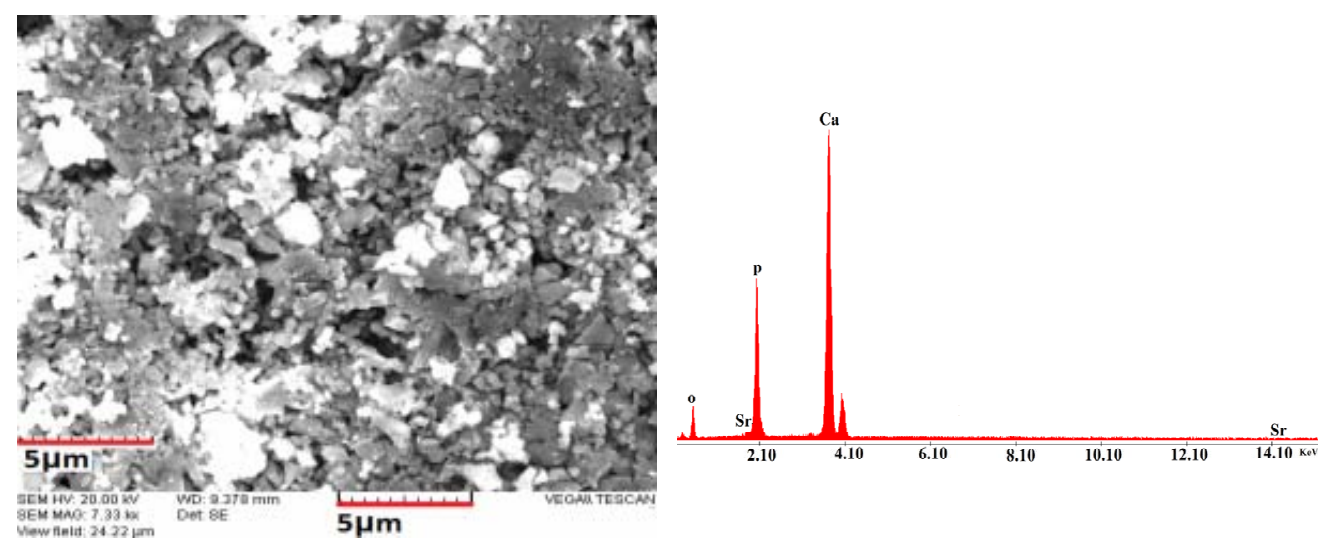

(a)

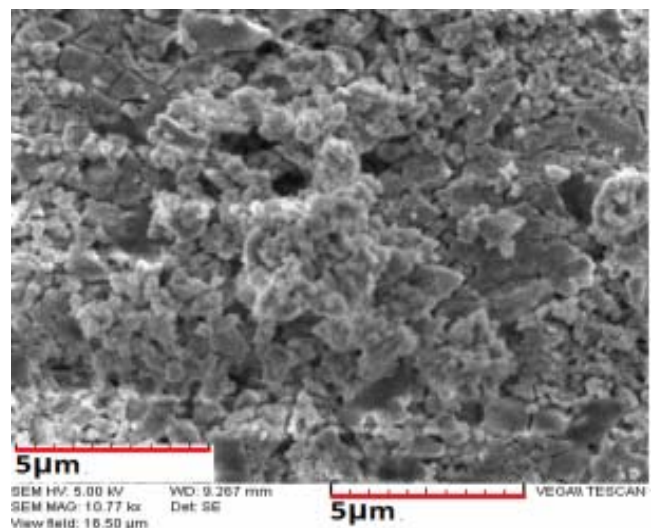

(b)

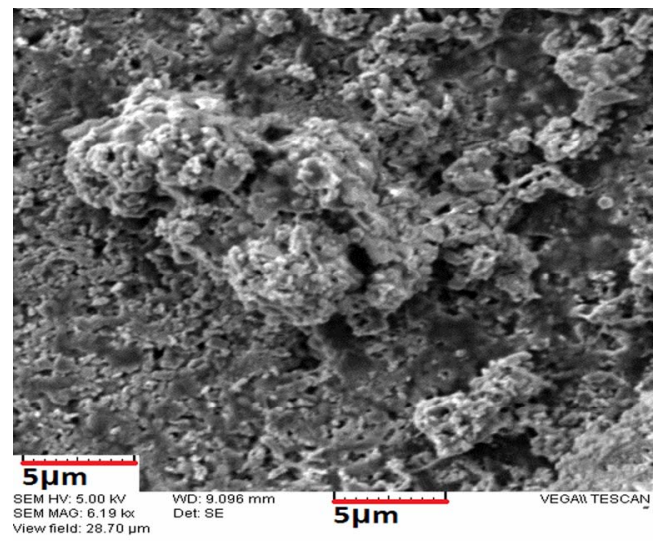

(d)

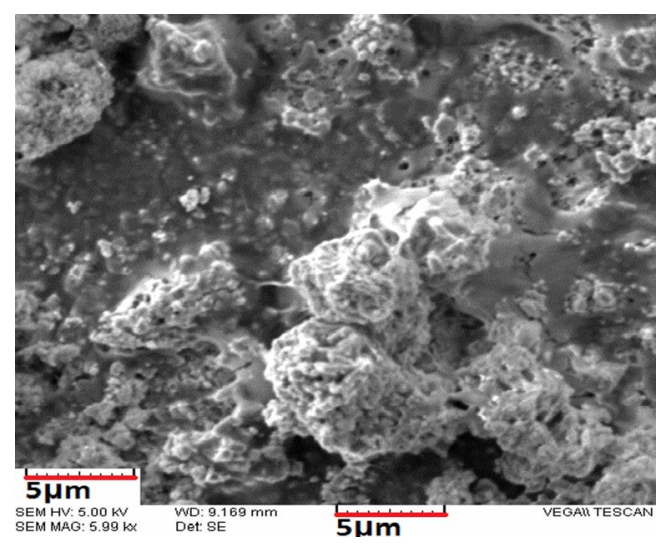

(c)

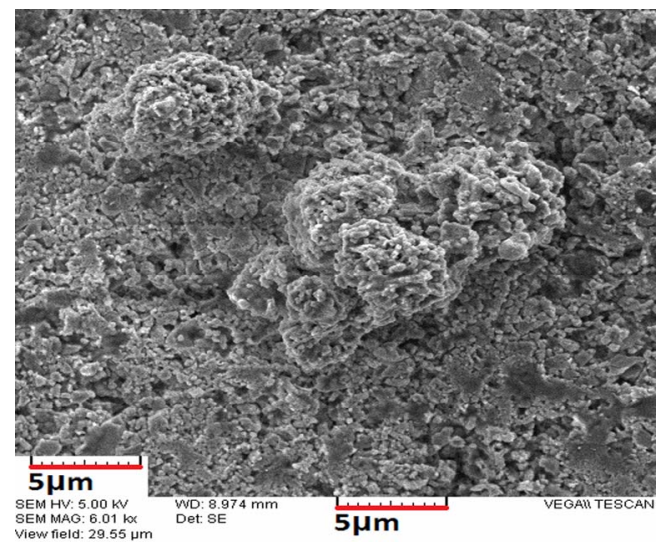

(e)

Figure 3. Scanning electron micrographs of Sr-HAp samples after soaking in m-SBF for certain durations. (a) Pristine Sr-Hap, EDAX of pristine Sr-Hap; (b) Sr-HAp 8d in m-SBF; (c) Sr-HAp 16d in m$\mathrm{SBF}$; (d) Sr-HAp 24d in m-SBF; (e) Sr-HAp 32d in m-SBF.

The Cole-Cole plots of Sr-HAp after particular period of incubation in m-SBF are depicted in Figure 5(b). All the incubated samples show similar kind of behaviour wherein a single semi-circle is observed. The shape of these semicircles does not change but size increases with increase in incubation period and the position of $Z_{\max }^{\prime \prime}$ slightly shifts to lower frequency side. This behaviour indubitably reveals the distribution of relaxation time instead of a single relaxation time in the material. Relaxation times for Sr-HAp samples, determined from the complex impedance graph, are found to be $232 \mu \mathrm{sec}, 371$ $\mu \mathrm{sec}, 371 \mu \mathrm{sec}, 750 \mu \mathrm{sec}$. This indicates that time required to depolarize the polarizing ions increases linearly with increase in incubating duration. The values of bulk resistance $\left(R_{b}\right)$ at different incubation duration have been obtained from the intercept of semicircular arcs on the 


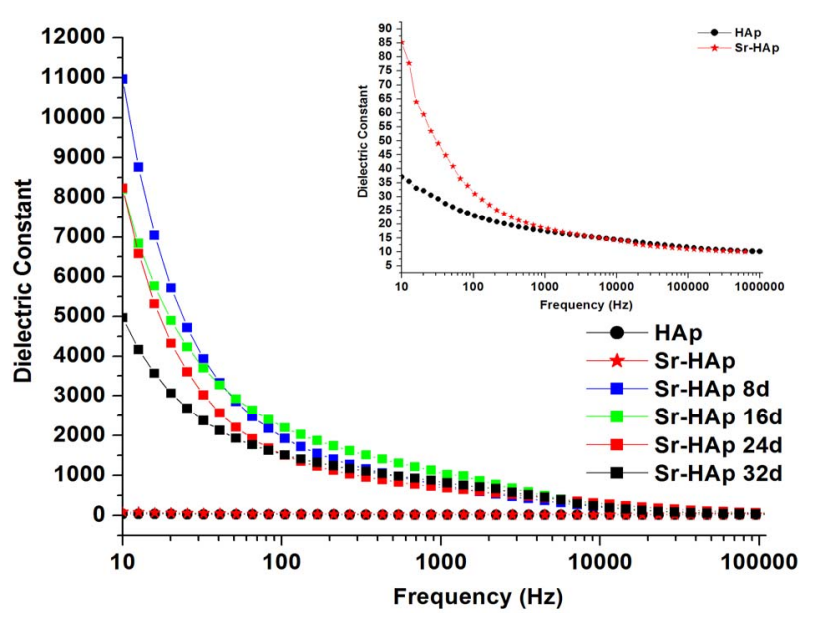

(a)

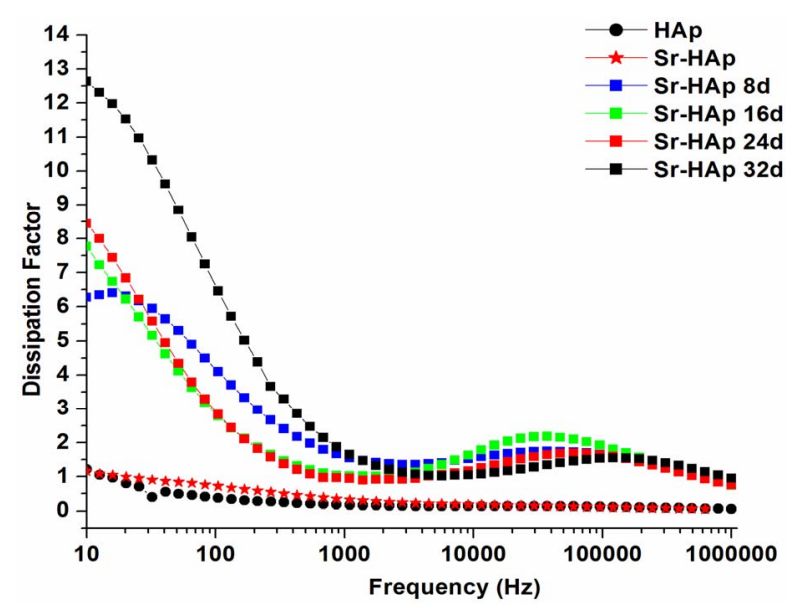

(b)

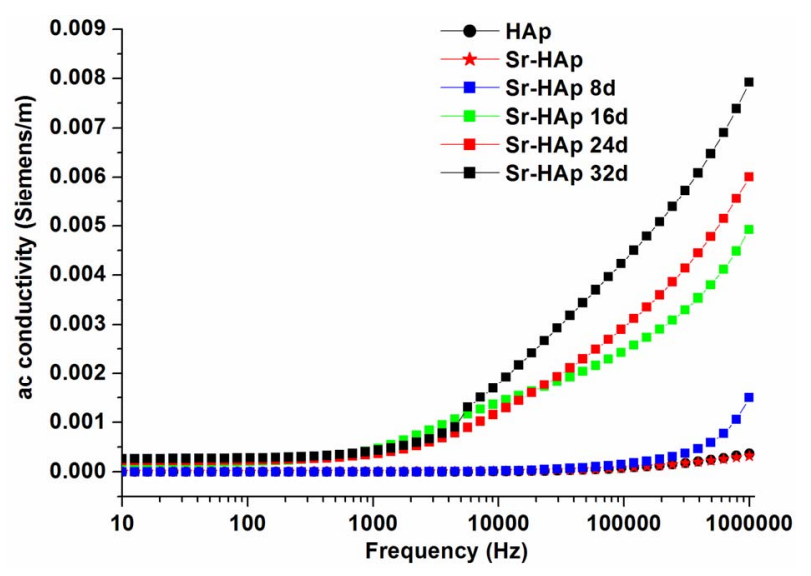

(c)

Figure 4. (a) Behavior of dielectric constant of hydroxyapatite with a frequency of applied ac field showing the dielectric dispersion; (b) The change in dielectric loss for hydroxyapatite, Sr-doped hydroxyapatite before incubation and incubated Srdoped hydroxyapatite with applied frequency; (c) Real part of ac conductivity as a function of applied frequency for hydroxyapatite, Sr-doped hydroxyapatite before incubation and Srdoped hydroxyapatite after incubation in modified SBF for 8 , $16,24,32$ days.

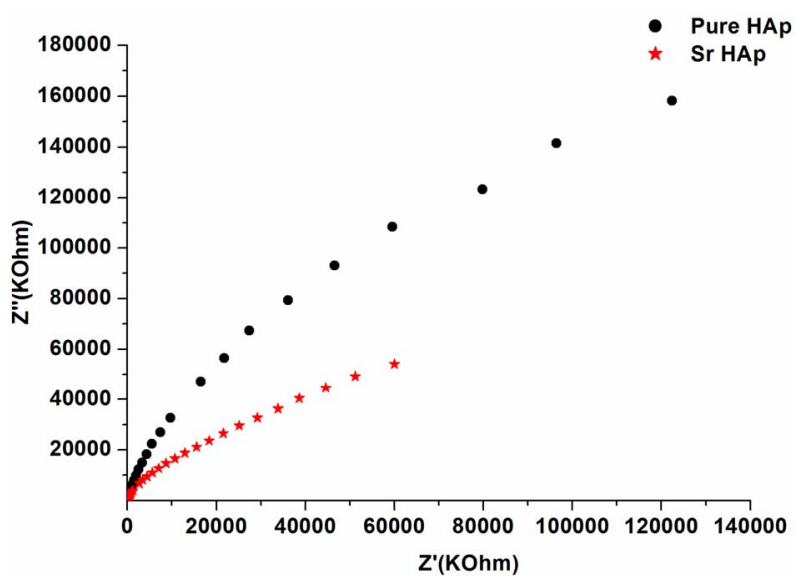

(a)

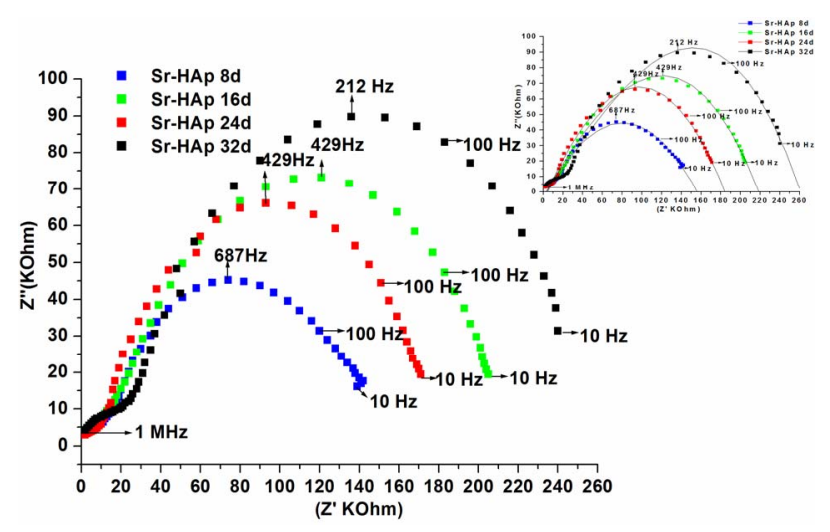

(b)

Figure 5. (a) Complex impedance plot of pure hydroxyapatite and strontium doped hydroxyapatite where $\left(-Z^{\prime \prime}\right)$ is plotted as positive where $Z^{\prime}, Z^{\prime \prime}$ are real and imaginary parts of impedance; (b) Complex impedance plot of strontium doped hydroxyapatite after incubation in $\mathrm{m}-\mathrm{SBF}$ as a function of incubation period.

real axis $\left(Z^{\prime}\right)$ and are given in the Table 3. It is observed that $R_{b}$ increases with rise in incubation period. The capacitance values $\left(C_{b}\right)$ are in the range of $\mathrm{nF}$.

Figure 6(a) shows the variation of $Z^{\prime}$ with frequency at different incubation periods. It is observed that the values of $Z^{\prime}$ decreases with increase in frequency and decrease in incubation period. This indicates that conductivity is increasing with increase in incubation period. For all variable incubation periods, the value of $Z^{\prime}$ merge together at higher frequencies. It reveals that dipoles are not able to follow high frequency fields. The variation of $Z^{\prime \prime}$ with frequency at various incubation periods is shown in Figure 6(b). The plot shows that $Z^{\prime \prime}$ values possess a maxima and position of this maxima shifts to lower frequency side with increase in incubation duration. This is related to relaxation process in HAp matrix. The magnitude of $Z^{\prime \prime}$ decreases with decrease in incubation period.

Figure 7 shows photoluminescence spectra of pure HAp and Sr-HAp without incubation along with incubated 
Table 3. Bulk resistances and capacitances of incubated SrHAp samples obtained by obtaining intercept of each semicircle on real axis from simulation.

\begin{tabular}{ccc}
\hline \multicolumn{3}{c}{ Bulk resistances and capacitances of incubated Sr-HAp samples } \\
\hline Incubated Sr-HAp samples & $R_{b}(\mathrm{kohm})$ & $C_{b}(\mathrm{nF})$ \\
\hline 8 days & 156 & 1.48 \\
16days & 219 & 1.69 \\
24 days & 184 & 2.01 \\
32 days & 260 & 2.88 \\
\hline
\end{tabular}

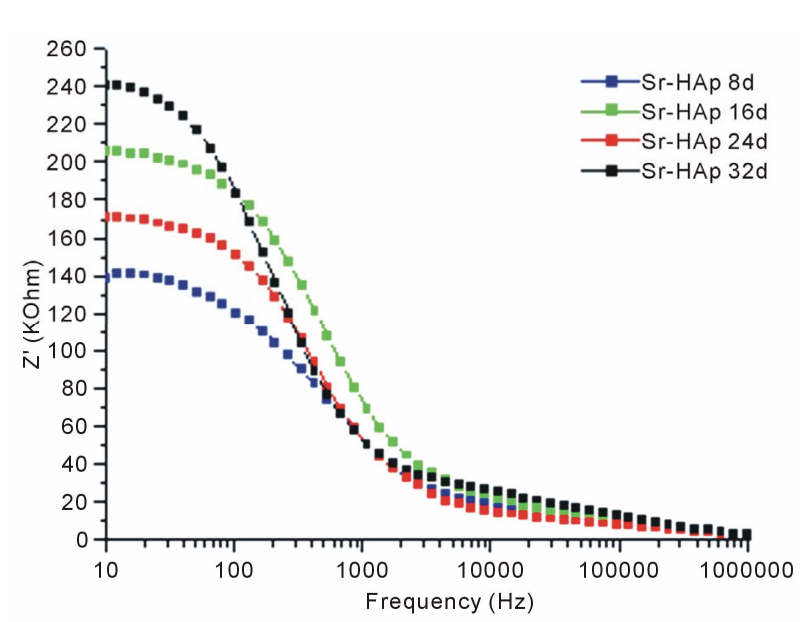

(a)

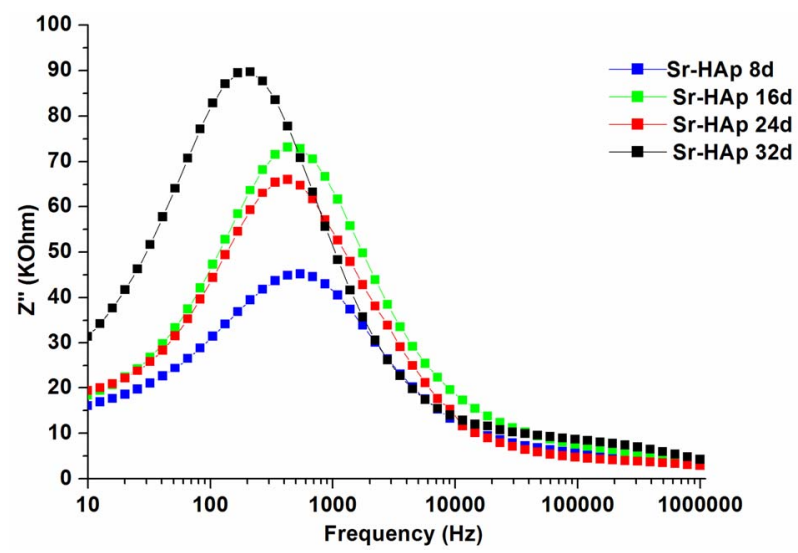

(b)

Figure 6. (a) Variation of real part of impedance $\left(Z^{\prime}\right)$ with frequency for m-SBF incubated Sr-HAp pellets; (b) Variation in imaginary part of impedance $\left(Z^{\prime \prime}\right)$ with frequency for Sr-HAp pellets after incubation in $\mathrm{m}-\mathrm{SBF}$.

Sr-HAp samples. The characteristic intense blue emission peaks are obvious in the emission spectra for all the samples. The results show that all HAp samples emit light of wavelength ranging from $409 \mathrm{~nm}-650 \mathrm{~nm}$ with a peak at around $476 \mathrm{~nm}$. There is essentially no shift in the spectral position for the characteristic emission peak, but the PL peak relative intensity changes with duration

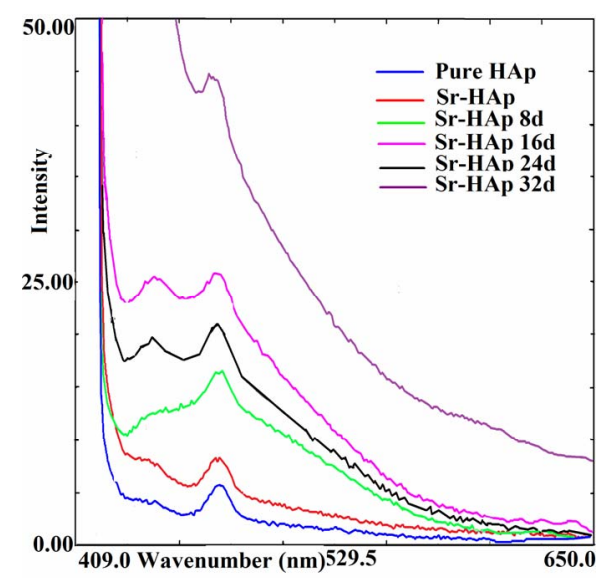

Figure 7. Photoluminescence spectra of hydroxyapatite samples showing intense blue emission at $476 \mathrm{~nm}$ for excitation at $409 \mathrm{~nm}$ showing the effect of incubation on emission intensity at room temperature.

of incubation in m-SBF along with appearance of an additional peak at around $445 \mathrm{~nm}$ after 16 days of incubation. Thus, it can be concluded that m-SBF incubated samples give more excellent PL performance than without incubated pure and Sr-HAp in terms of PL intensity. The 32 days incubated Sr-HAp has the highest PL intensity in the six samples. It may be due to growth of apatite layer during incubation in $\mathrm{m}-\mathrm{SBF}$.

\section{DISCUSSION}

The formation of biological apatite layer on the bioactive Sr-HAp surface takes place via dissolution and precipitation processes. In the Sr-HAp samples, $\mathrm{Ca}^{2+}$ ions are partially replaced by $\mathrm{Sr}^{2+}$ ions using ion exchange process. This partial replacement of $\mathrm{Ca}^{2+}$ ions by $\mathrm{Sr}^{2+}$ ions in HAp matrix is responsible for increase in solubility of HAp as a result of lattice expansion due to higher atomic radius of $\mathrm{Sr}^{2+}$ ion than $\mathrm{Ca}^{2+}$ ion. Due to dissolution of Sr-HAp, when immersed in $\mathrm{m}-\mathrm{SBF}, \mathrm{Ca}^{2+}$ and $\mathrm{Sr}^{2+}$ ions are released. This increases the ionic activity product of apatite in the surrounding body fluid. According to kinetics of bone like apatite formation, the $\left(\mathrm{PO}_{4}^{3-}\right)$ hydroxyapatite surface charges interact with the $\mathrm{Ca}^{2+}$ ions in the m-SBF fluid to form calcium reach surface. This leads to increase in positive surface charge of hydroxyapatite. This calcium rich surface further interacts with the $\left(\mathrm{PO}_{4}^{3-}\right)$ ions from the body fluid [46]. Thus, migration of calcium and phosphate ions to the surface readily induces apatite precipitation on the HAp surface to get stabilized by being crystallized into bonelike apatite. They grow further consuming calcium and phosphate ions from the simulated body fluid spontaneously. Thus, during apatite layer formation various ions play a key role because ionic interaction takes place between sur- 
face ions and ions from the $\mathrm{m}$-SBF.

Generally in most of the ceramics, the major role is of ionic polarization along with some electronic contribution. The Sr-HAp structure contains the ions like $\mathrm{Sr}^{2+}$, $\mathrm{Ca}^{2+}, \mathrm{PO}_{4}^{3-}$ and $(\mathrm{OH})^{-}$. Being heavy and less mobility, the ionic displacement begins to lag the field reversals with the increase changes and ionic polarization decreases. Therefore, the dielectric constant decreases as frequency of applied field increases. This results in increasing the loss factor and contributing less to the dielectric constant. At still higher frequencies, the field reversals are such that the ions cannot cope with these changes.

Relaxation time is the time required for the reorientation of the ions to their original position while the ac field is applied. When the ions are not capable to cope up with ac field changes, dielectric constant decreases. Increase in immersion time leads to increase in relaxation time. The relaxation time for a sample of 8 days incubation in $\mathrm{m}-\mathrm{SBF}$ is observed to be least compared to other samples which are incubated for longer durations. Therefore, it is concluded that ions in a sample with 8 days incubation can reorient themselves quickly compared those for higher incubated samples. Therefore, the dielectric constant is observed to be the highest for 8 days incubated sample and goes on decreasing with incubation period.

Furthermore, the condictivity is associated to the ionic mobility of surface-ionized species. Therefore, the increase of conductivity with immersion period may be probably associated to a change of the nature of the surface sites after incubation. It is believed that the immersion process provides enough water conditions, which accelerates the hydration rate of Sr-HAp. With the immersion proceeding in the early stages, the dissolution provides favorable sites for the formation of apatite nuclei. Then, as the immersion time increases in the growth stage, $\mathrm{Ca}$ and $\mathrm{P}$ concentrations decreases gradually probably due to the Ca-P formation by consuming the calcium and phosphate ions in m-SBF which might be affecting on conductivity.

The AC conductivity in a dielectric material is due to hopping mechanism of bound charges due to absence of free charge carriers in such materials. These bound charges hop back and forth from one bound state to another. The hopping mechanism is well supported by the conduction behavior of the samples wherein AC conductivity increases at higher frequencies.

The $\mathrm{Ca}^{2+}$ or $\mathrm{Sr}^{2+}$ ions and the $\mathrm{PO}_{4}^{3-}$ groups are not responsible for luminescence phenomenon in HAp. Therefore, the observed luminescence from pure and Srdoped HAp samples must be due to some impurities in the host lattice. The FT-IR spectrum shows the presence of carbonate ions at 1440 and $880 \mathrm{~cm}^{-1}$. This indicates the existence of some carbon-related impurities on the surface of the HAp samples. The impurities may be due to the carbonate inclusion during the growth process in hydroxyapatite lattice and is responsible for the selfactivated luminescence.

\section{ACKNOWLEDGEMENTS}

This work is carried out under Major Research Project (F.No.36-241/ 2008 (SR)) sanctioned by University Grants Commission, New Delhi.

\section{REFERENCES}

[1] Gu, Y.W., Khor, K.A. and Cheang, P. (2004) Bone-like apatite layer formation on hydroxyapatite prepared by spark plasma sintering (SPS). Biomaterials, 25, 41274134. doi:10.1016/i.biomaterials.2003.11.030

[2] Ni, J. and Wang, M. (2002) In vitro evaluation of hydroxyapatite reinforced polyhydroxybutyrate composite. $\mathrm{Ma}$ terials Science and Engineering: C, 20, 101-109. doi:10.1016/S0928-4931(02)00019-X

[3] Sánchez-Salcedo, S., Balas, F., Izquierdo-Barba, I. and Vallet-Regi, M. (2009) In vitro structural changes in porous HA/b-TCP scaffolds in simulated body fluid. Acta Biomaterialia, 5, 2738-2751. doi:10.1016/j.actbio.2009.03.025

[4] Pecheva, E.V., Pramatarova, L.D., Maitz, M.F., Pham, M.T. and Kondyuirin, A.V. (2004) Kinetics of hydroxyapatite deposition on solid substrates modified by sequential implantation of $\mathrm{Ca}$ and $\mathrm{P}$ ions. Part II: Morphological, composition and structure study. Applied Surface Science, 235, 170-175. doi:10.1016/j.apsusc.2004.05.178

[5] Chavan, P.N., Bahir, M.M., Mene, R.U., Mahabole, M.P. and Khairnar, R.S. (2010) Study of nanobiomaterial hydroxyapatite in simulated body fluid: Formation and growth of apatite. Materials Science and Engineering: B, 168, 224-230. doi:10.1016/j.mseb.2009.11.012

[6] Arnich, N., Marie-Claire, L., Laurensot, F., Podor, R. Montiel, A. and Burnel D. (2003) In vitro and in vivo studies of lead immobilization by synthetic hydroxyapatite. Environmental Pollution, 124, 139-149. doi:10.1016/S0269-7491(02)00416-5

[7] Wong, C.T., Lu, W.W., Chan, W.K., Cheung, K.M.C., Luk, K.D.K., Lu, D.S., Rabie, A.B.M., Deng, L.F. and Leong, J.C.Y. (2004) In vivo cancellous bone remodeling on a strontium containing hydroxyapatite (Sr-HA) bioactive cement. Journal of Biomedical Materials Research Part A, 68, 513-521. doi:10.1002/jbm.a.20089

[8] Labella, R., Braden, M. and Debt, S. (1994) Novel hydroxyapatite-based dental composites. Biomaterials, 15, 1197-1200. doi:10.1016/0142-9612(94)90269-0

[9] Werber, R., Klaus-Dieter, Brauer, R.B., Weiß, W. and Becker, K. (2000) Osseous integration of bovine hydroxyapatite ceramic in metaphyseal bone defects of the distal. The Journal of Hand Surgery, 25A, 833-841. doi:10.1053/jhsu.2000.16354

[10] Mahabole, M.P., Aiyer, R.C., Ramakrishna, C.V., Sreedhar, B. and Khairnar, R.S. (2005) Synthesis, characteriza- 
tion and gas sensing property of hydroxyapatite ceramic. Bulletin of Materials Science, 28, 535-545. doi:10.1007/BF02706339

[11] Mene, R.U., Mahabole, M.P., Aiyer, R.C. and Khairnar, R.S. (2010) Hydroxyapatite nano-ceramic thick film: An efficient $\mathrm{CO}_{2}$ gas sensor. The Open Applied Physics Journal, 3, 10-16.

[12] Mene, R.U., Mahabole, M.P. and Khairnar, R.S. (2011) surface modified hydroxyapatite thick films for $\mathrm{CO} 2$ gas sensing application: Effect of swift heavy ion irradiation. Radiation Physics and Chemistry, 80, 682-687. doi:10.1016/j.radphyschem.2011.02.002

[13] Furuta, S., Katsuki, H. and Komarneni, S. (2000) Removal of lead ions using porous hydroxyapatite monoliths synthesized from gypsum waste. Journal of Ceramic Society of Japan, 108, 315-317. doi:10.2109/jcersj.108.1255 315

[14] Aizawa, M., Howell, S., Itatani, K., Yokogawa, Y., Nishizawa, K., Toriyama, M. and Kameyama, T. (2000) Fabrication of porous ceramics with well-controlled open pores by sintering of fibrous hydroxyapatite particles. Journal of Ceramic Society of Japan, 108, 249-253. doi:10.2109/jcersj.108.1255 249

[15] Sugiyama, S., Nakanishi, T., Ishimura, T., Moriga, T., Hayashi, H., Shigomoto, N. and Moffat, J.B. (1999) Preparation characterization and thermal stability of lead hydroxyapatite. Journal of Solid State Chemistry, 143, 296302. doi:10.1006/jssc.1998.8126

[16] Schroder, E., Jonsson, T. and Poole L. (2003) Hydroxyapatite chromatography: Altering the phosphate-dependent elution profile of protein as a function of $\mathrm{pH}$. Analytical Biochemistry, 313, 176-178. doi:10.1016/S0003-2697(02)00567-5

[17] Park, Y.S. and Yamazaki, Y. (2005) Novel Nafion/Hydroxyapatite composite membrane with high crystallinity and low methanol crossover for DMFCs. Polymer Bulletin, 53, 181-192. doi:10.1007/s00289-004-0310-0

[18] Quilitz, M., Steingröver, K. and Veith, M. (2010) Effect of the $\mathrm{Ca} / \mathrm{P}$ ratio on the dielectric properties of nanoscaled sub stoichiometric hydroxyapatite. Journal of Materials Science: Materials in Medicine, 21, 399-405. doi:10.1007/s10856-009-3875-1

[19] Shi, S.L., Pan, W., Han, R.B. and Wan, C.L. (2006) Electrical and dielectric behaviours of $\mathrm{Ti}_{3} \mathrm{SiC}_{2}$ /hydroxyapatite composites. Applied Physics Letters, 88, 052903. doi:10.1063/1.2168684

[20] Gittings, J.P., Bowen, C.R., Dent, A.C.E., Turner, I.G., Baxter, F.R. and Chaudhuri, J.B. (2009) Electrical characterization of hydroxyapatite-based bioceramics. Acta Biomaterialia, 5, 743-754. doi:10.1016/j.actbio.2008.08.012

[21] Silva, C.C., Almeida, A.F.L., De oliveira, R.S., Pinheiro, A.G., Goes, J.C. and Sombra, A.S.B. (2003) Dielectric permittivity and loss of hydroxyapatite screen-printed thick films. Journal of Materials Science, 38, 3713-3720. doi:10.1023/A:1025963728858

[22] Hoepefner, T.P. and Case, E.D. (2002) The porosity dependence of the dielectric constant for sintered hydroxyapatite. Journal of Biomedical Materials Research, 60,

\section{3-650. doi:10.1002/jbm.10131}

[23] Ikoma, T., Yamazaki, A., Nakamura, S. and Akao, M. (1999) Preparation and dielectric property of sintered monoclinic hydroxyapatite. Journal of Materials Science Letters, 18, 1225-1228. doi:10.1023/A:1006610521173

[24] Laghzizil, A., Elherch, N., Bouhaouss, A., Lorente, G., Coradin, T. and Livage, J. (2001) Electrical behavior of hydroxyapatites $\mathrm{M}_{10}\left(\mathrm{PO}_{4}\right)_{6}(\mathrm{OH})_{2}(\mathrm{M}=\mathrm{Ca}, \mathrm{Pb}, \mathrm{Ba}) . M a$ terials Research Bulletin, 36, 953-962. doi:10.1016/S0025-5408(01)00576-1

[25] Ni, G.X., Lin, J.H., Chiu, P.K.Y., Li, Z.Y. and Lu, W.W. (2010) Effect of strontium-containing hydroxyapatite bone cement on bone remodeling following hip replacement. Journal of Materials Science: Materials in Medicine, 21, 377-384. doi:10.1007/s10856-009-3866-2

[26] Saint-Jean, J., Camire, C.L., Nevsten, P., Hansen, S. and Ginebra, M.P. (2005) Study of the reactivity and in vitro bioactivity of Sr-substituted $\alpha$-tcp cements. Journal of Materials Science: Materials in Medicine, 16, 993-1001. doi:10.1007/s10856-005-4754-Z

[27] Guo, D., Xu, K., Zhao, X. and Han, Y. (2005) Development of a strontium-containing hydroxyapatite bone cement. Biomaterials, 26, 4073-4083. doi:10.1016/j.biomaterials.2004.10.032

[28] Kanno, T., Horiuchi, J.I., Koba, M., Motogami, Y. and Akazawa, T. (1999) Characteristics of the carbonate ions incorporated into calcium, partially-strontium-substituted and strontium apatites. Journal of Materials Science Letters, 18, 1343-1345. doi:10.1023/A:1006638416515

[29] Wang, X. and Ye, J. (2008) Variation of crystal structure of hydroxyapatite in calcium phosphate cement by the substitution of strontium ions. Journal of Materials Science: Materials in Medicine, 19, 1183-1186. doi:10.1007/s10856-007-3209-0

[30] Bera, J., Kalia, V. and Roy, P.K. (2004) Comparison of electrical properties between $\mathrm{Ca}$ and $\mathrm{Sr}$ hydroxyapatite materials. International Symposium on Advanced Materials and Processing, 6-8 December 2004, Indian Institute of Technology (IIT), Kharagpur.

[31] Ternane, R., Trabelsi-Ayedi, M., Kbir-Ariguib, N. and Piriou, B. (1999) Luminescent properties of $\mathrm{Eu}^{3+}$ in calcium hydroxyapatite. Journal of Luminescence, 81, 165170. doi:10.1016/S0022-2313(98)00172-0

[32] Chen, F., Zhu, Y.J., Zhang, K.H., Wu, J., Wang, K.W., Tang, Q.L. and Mo, X.M. (2011) Europium-doped amorphous calcium phosphate porous nanospheres: Preparation and application as luminescent drug carriers. Nanoscale Research Letters, 6, 67. doi:10.1186/1556-276X-6-67

[33] Chen, F., Huang, P., Zhu, Y.J., Wu, J., Zhang, C.L. and Xiang Cui, D.X. (2011) The photoluminescence, drug delivery and imaging properties of multifunctional $\mathrm{Eu}^{3+}$ / $\mathrm{Gd}^{3+}$ dual-doped hydroxyapatite nanorods. Biomaterials, 32, 9031-9039. doi:10.1016/j.biomaterials.2011.08.032

[34] Silva, C.C., Filho, F.P., Sombra, A.S.B., Rosa, I.L.V., Leite, E.R., Longa, E. and Varela, J.A. (2008) Study of structural and photoluminescence properties of $\mathrm{Ca}_{8} \mathrm{Eu}_{2}\left(\mathrm{PO}_{4}\right)_{6} \mathrm{O}_{2}$. Journal of Fluorescence, 18, 253-259. 
doi:10.1007/s10895-007-0242-9

[35] Graeve, O.A., Raghunath, K., Madadi, A., Brandon, C.W. and Glass, K.C. (2010) Luminescence variations in hydroxyapatites doped with $\mathrm{Eu}^{2+}$ and $\mathrm{Eu}^{3+}$ ions. Biomaterials, 31, 4259-4267. doi:10.1016/j.biomaterials.2010.02.009

[36] Zhang, C.M., Yang, J., Quan, Z.W., Yang, P.P., Li, C.X., Hou, Z.Y. and Lin J. (2009) Hydroxyapatite nano and microcrystals with multiform morphologies: Controllable synthesis and luminescence properties. Crystal Growth and Design, 9, 2725-2733. doi:10.1021/cg801353n

[37] Muller, F.A., Muller, L., Zollfrank, C. and Greil, P. (2006) Inherent luminescence of annealed biomimetic apatites. Engineering Materials, 311, 655-658.

[38] Chung, R.J., Chin, T.S., Cheng, H.Y., Wen, H.W. and Hsieh, M.F. (2007) Photo-luminescent hydroxyapatite coating through a bio-mimetic process. Biomolecular Engineering, 24, 459-461. doi:10.1016/j.bioeng.2007.07.006

[39] Zhang, C., Cheng, Z., Yang, P., Xu, Z., Peng, C., Li, G. and Lin J. (2009) Architectures of strontium hydroxyapatite microspheres: Solvothermal Synthesis and Luminescence properties. Langmuir, 25, 13591-13598. doi:10.1021/la9019684

[40] Zhang, C., Li, C., Huang, S., Hou, Z., Cheng, Z., Yang, P., Peng, C. and Lin J. (2010) Self-activated luminescent and mesoporous strontium hydroxyapatite nanorods for drug delivery. Biomaterials, 31, 3374-3383. doi:10.1016/j.biomaterials.2010.01.044

[41] Tas, A.C. (2000) Synthesis of biomimetic Ca-hydroxya- patite powders at $37^{\circ} \mathrm{C}$ in synthetic body fluids. Biomaterials, 21, 1429-1438. doi:10.1016/S0142-9612(00)00019-3

[42] Jalota, S., Bhaduri, S.B. and Tas, A.C. (2008) Using a synthetic body fluid (SBF) solution of $27 \mathrm{mM} \mathrm{HCO}_{3}^{-}$to make bone substitutes more osteointegrative. Material Science and Engineering C, 28, 129-140. doi:10.1016/j.msec.2007.10.058

[43] Gittings, J.P., Bowen, C.R., Turner, I.G., Dent, A.C.E., Baxter, F.R. and Chaudhuri, J.B. (2008) Dielectric properties of hydroxyapatite based ceramics. In: Dimov, S. and Menz, W. Eds., Multi Material Micro Manufacture, Research Publishing, Cardiff, 2-5.

[44] Gittings, J.P., Bowen, C.R., Turner, I.G., Baxter, F.R. and Chaudhuri, J.B. (2007) Characterization of ferroelectric calcium phosphate composites and ceramics. European Ceramic Society, 27, 4187-4190. doi:10.1016/j.jeurceramsoc.2007.02.120

[45] Silva, C.C., Graca, M.P.F., Sombra, A.S.B. and Valente, M.A. (2009) Structural and electrical study of calcium phosphate obtained by a microwave radiation assisted procedure. Physica B: Condensed Matter, 404, 1503-1508. doi:10.1016/j.physb.2009.01.015

[46] Hyun-Min, K., Teruyuki, H., Kokubo, T. and Takashi, N. (2005) Process and kinetics of bonelike apatite formation on sintered hydroxyapatite in a simulated body fluid. Biomaterials, 26, 4366-4373.

doi:10.1016/j.biomaterials.2004.11.022 